\title{
A NONLINEAR INTEGRAL EQUATION OCCURRING IN A SINGULAR FREE BOUNDARY PROBLEM ${ }^{1}$
}

\author{
BY \\ KLAUS HOLLLIG ${ }^{2}$ AND JOHN A. NOHEL
}

ABSTRACT. We study the Cauchy problem

$$
\left\{\begin{array}{l}
u_{t}=\phi\left(u_{x}\right)_{x}, \quad(x, t) \in \mathbf{R} \times \mathbf{R}_{+}, \\
u(\cdot, 0)=f
\end{array}\right.
$$

with the piecewise linear constitutive function $\phi(\xi)=\xi_{+}=\max (0, \xi)$ and with smooth initial data $f$ which satisfy $x f^{\prime}(x) \geqslant 0, x \in \mathbf{R}$, and $f^{\prime \prime}(0)>0$. We prove that free boundary $s$, given by $u_{x}\left(s(t)^{+}, t\right)=0$, is of the form

$$
s(t)=-\kappa \sqrt{t}+o(\sqrt{t}), \quad t \rightarrow 0^{+},
$$

where the constant $\kappa=0.9034 \ldots$ is the (numerical) solution of a particular nonlinear equation. Moreover, we show that for any $\alpha \in(0,1 / 2)$,

$$
\left|\frac{d^{2}}{d t^{2}} f(s(t))\right|=O\left(t^{\alpha-1}\right), \quad t \rightarrow 0^{+} .
$$

The proof involves the analysis of a nonlinear singular integral equation.

1. Introduction and result. We study the Cauchy problem

$$
\left\{\begin{array}{l}
u_{t}=\phi\left(u_{x}\right)_{x}, \quad(x, t) \in \mathbf{R} \times \mathbf{R}_{+}, \\
u(\cdot, 0)=f
\end{array}\right.
$$

with the piecewise linear constitutive function $\phi: \mathbf{R} \rightarrow \mathbf{R}_{+}$given by $\phi(\xi)=\xi_{+}=$ $\max (\xi, 0)$; the initial data $f: \mathbf{R} \rightarrow \mathbf{R}$ are assumed smooth, specifically $f \in C^{3}(\mathbf{R})$ with bounded derivatives, and satisfy the conditions

$$
\left\{\begin{array}{l}
x f^{\prime}(x) \geqslant 0, \quad x \in \mathbf{R}, \\
f^{\prime \prime}(0)>0 .
\end{array}\right.
$$

Our motivation for the study of the Cauchy problem (1), (2) is its similarity to the well-known one-phase Stefan problem (in one space dimension) $[\mathbf{3}, \mathbf{4}, \mathbf{7}, \mathbf{8}]$ in which one would assume $f^{\prime}(x) \equiv-1$ for $x<0$, as well as $f^{\prime}(x)>0$ for $x>0$, so that $f^{\prime}$ has a jump discontinuity at $x=0$. The assumption (2) yields a different behavior of

Received by the editors March 11, 1983. Presented to the Society at its 808th meeting in Evanston, November 12, 1983.

1980 Mathematics Subject Classification: Primary 35K55, 35K65, 45G05.

Key words and phrases. Cauchy problem, parabolic, nonlinear, free boundary regularity, nonlinear singular integral equation.

'Sponsored by the United States Army under Contract No. DAAG29-80-C-0041.

${ }^{2}$ This material is based upon work partially supported by the National Science Foundation under Grant No. MCS-7927062, Mod. 2. 
the solution $u$ and of the resulting free boundary. Indeed, here (cf. the Theorem below), the free boundary $s$, given by $u_{x}\left(s(t)^{+}, t\right)=0$, is of the form

$$
s(t)=-\kappa \sqrt{t}+O\left(t^{1 / 2+\alpha}\right), \quad t \rightarrow 0^{+},
$$

where $\kappa$ is a positive constant and $0<\alpha<1 / 2$. Thus, the function $s$ is not (infinitely) differentiable at $t=0$, contrary to the situation for the Stefan problem [7].

The result (3) is established by solving a nonlinear integral equation (15) with kernels which depend on the unknown function $s$ and which are also singular in the sense that the integral on $(0, t)$ of the kernel does not approach zero as $t \rightarrow 0^{+}$. One consequence of this is that the integral operator defined by (15) is not compact in a suitable Hölder class.

The principal motivation for the study of the Cauchy problem (1), (2) is that it serves as a prototype of nonlinear parabolic problems which arise as monotone "convexifications" of nonlinear diffusion equations with nonmonotone constitutive functions $\phi$ (see [5 and 6]); in [6, §4] the reader will also find the formulation and preliminary analysis of such a convexified problem, corresponding to a piecewise linear nonmonotone $\phi$ (specifically, $\phi^{\prime}((-\infty, a) \cup(b, \infty))>0, \phi^{\prime}(a, b)<0,0<a<$ $b<\infty)$. The analysis in [5] shows the existence the infinitely many solutions $u$ of the nonmonotone problem, each having $u_{x}$ bounded, and $u_{x}$ omitting values in $[a, b]$; thus each solution $u$ exhibits phase changes. Numerical experiments further suggest the conjecture that the "physically correct" solution of the nonmonotone problem is the one which, as $t \rightarrow \infty$, approaches the unique solution of the appropriately related convexified monotone problem. However, for small $t>0$ the behavior of the solution of (1), (2) is qualitatively different (see (3)). The present study of (1), (2) is intended as a step towards the understanding of this intriguing phenomenon. The relation of the convexified problem in [6] to the Cauchy problem (1), (2) is clear (the particular boundary conditions in [6] do not play a role in the analysis of the free boundary curve).

It is simple to give a formal explanation for (3). We rewrite (1), (2) as the free boundary problem

$$
\left\{\begin{array}{l}
u_{t}=u_{x x}, \quad s(t)<x<\infty, \quad t \in \mathbf{R}_{+}, \\
u_{x}(s(t), t)=0 \\
u(\cdot, 0)=f
\end{array}\right.
$$

From the constitutive function $\phi$ one also has the equation

$$
\left\{\begin{array}{l}
u_{t}(x, t)=0, \quad-\infty<x<s(t), \quad t \in \mathbf{R}_{+}, \\
u(\cdot, 0)=f .
\end{array}\right.
$$

Therefore, assuming the continuity of $u$ across the free boundary $s(t)$ and assuming that $s$ is monotone decreasing (cf. paragraph preceding the Theorem), we have

$$
\left\{\begin{array}{l}
u(s(t), t)=f(s(t)), \quad t \in \mathbf{R}_{+}, \\
s(0)=0 .
\end{array}\right.
$$


Differentiating (5) with respect to $t$ and using $u_{x}\left(s(t)^{+}, t\right)=0$, where " + " denotes the limit from the right, we obtain

$$
f^{\prime}(s(t)) s^{\prime}(t)=u_{x x}\left(s(t)^{+}, t\right) .
$$

Since by the assumption (2)

$$
f^{\prime}(x)=f^{\prime \prime}(0) x+O\left(|x|^{2}\right), \quad|x| \rightarrow 0^{+},
$$

a simple calculation formally yields (3) with $\kappa=\sqrt{2}$ (provided one assumes continuity from the right of $u_{t}$ and $u_{x x}$ up to the free boundary $s$ ).

The rigorous treatment of the problem consists of analyzing, in $\S 3$, the nonlinear integral equation (15) for the free boundary $x=s(t)$. Our analysis shows that (3) holds, but that the constant $\kappa$ is the solution of the nonlinear equation (16); its numerical value is $\kappa=0.9034 \ldots$, and not $\kappa=\sqrt{2}$ which was predicted by the above formal calculation. It also follows that $s(t)$ is smooth for $t>0$ thus justifying (5) and (6) for positive $t$; in particular, one sees from (6) that $s$ is as smooth as the initial function $f$ is. We remark that for $t \geqslant \varepsilon>0$ the problem (1), (2) can also be viewed as a one-phase Stefan problem; consequently, the results in Kinderlehrer and Nirenberg [7] yield the regularity of the free boundary for $t>0$.

The existence of a unique generalized continuous solution for problem (1), and hence of a unique free boundary, follows from nonlinear semigroup theory for $m$-accretive operators $[\mathbf{1}, 2]$. Approximating (1) by an implicit Euler scheme, one can also show the existence of the free boundary $s$ which is Hölder continuous on $[0, \infty)$ with exponent $1 / 2$ and monotone decreasing. However, using such general methods, it is not possible to analyze the precise behavior of $s$ at $t=0$.

Our main result is

THEOREM. Define

$$
r(t)=d f(s(t)) / d t
$$

Then for any $\alpha \in(0,1 / 2)$ there exists $T>0$ such that $r$ is continuous on $[0, T]$ and satisfies

$$
t^{1-\alpha}\left|r^{\prime}(t)\right| \leqslant c(f), \quad 0<t \leqslant T,
$$

where $c(f)>0$ is a constant which depends on the data $f$. Moreover, (3) holds with

$$
\kappa:=\left(2 \frac{r(0)}{f^{\prime \prime}(0)}\right)^{1 / 2}=0.9034 \ldots
$$

The constant $\mathrm{\kappa}$ is the (numerical) solution of equation (16) in $\$ 3$.

By the definition of $\kappa$, the result (3) follows from (7) and the assertion (8). To see this, we solve (7) for $s$. Let $R(t)=\int_{0}^{t} r(\tau) d \tau$ and integrate (7), obtaining

$$
R(t)=f(s(t))-f(0) \text {. }
$$

Define the function $g$ implicitly by

$$
g(-\operatorname{sign}(x) \sqrt{f(x)-f(0)})=x .
$$


Since we assume that

$$
f(x)-f(0)=\beta^{2} x^{2}+O\left(|x|^{3}\right), \quad|x| \rightarrow 0
$$

$\left(\beta^{2}=f^{\prime \prime}(0) / 2\right), g$ is well defined for small $|x|$ and

$$
g(x)=-\beta^{-1} x+O\left(|x|^{2}\right), \quad|x| \rightarrow 0 .
$$

For a small interval $[0, T]$, the monotone decreasing solution of $(7)$ is given by

$$
s(t)=g(\sqrt{R(t)}), \quad 0 \leqslant t \leqslant T,
$$

and (3) folows from (8) and (10).

The Theorem describes the regularity of the free boundary at $t=0$. It is sharp in the sense that, unless $f^{\prime \prime \prime}(0)=0$, the estimate (8) does not hold for $\alpha>1 / 2$ (cf. the Remark at the end of $\S 3$ ).

It should also be observed that the second derivatives of the solution $u$ are not continuous at the point $(x, t)=(0,0)$, because using $(6),(7)$ and the definition of $\kappa$ one has

$$
\lim _{t \rightarrow 0^{+}} u_{x x}\left(s(t)^{+}, t\right)=r(0)=\frac{\kappa^{2}}{2} f^{\prime \prime}(0) \neq \lim _{x \rightarrow 0^{+}} u_{x x}(x, 0)=f^{\prime \prime}(0) .
$$

However, on the set $\left\{t: f^{\prime}(s(t))<0\right\}$ the free boundary $s$ is as smooth as the function $f$. This can be shown by a bootstrap argument, using standard regularity results for the heat equation on a domain with curved boundaries. We believe that the Theorem can be extended to a general monotone constitutive function $\phi$ with $\phi^{\prime}$ discontinuous at 0 and with $\phi^{\prime}(\xi) \geqslant c>0, \xi \in \mathbf{R}_{+}$; the corresponding value of $\kappa$ will depend on $\phi^{\prime}\left(0^{+}\right)$.

The Theorem is proved in $\S 3$ by solving an integral equation for the function $r$ derived in $\$ 2$.

We are grateful for helpful discussions with our colleagues Tom Beale, Carl de Boor, Michael Crandall and Emmanuel DiBenedetto; we also thank Fred Sauer for the numerical computations.

2. The integral equation for the free boundary. Let

$$
\Gamma(x, t):=\frac{1}{2 \sqrt{\pi}} t^{-1 / 2} \exp \left(-\frac{x^{2}}{4 t}\right)
$$

denote the fundamental solution of the heat equation. Let $v:=u_{x}$ be the solution of the problem

$$
\left\{\begin{array}{l}
v_{t}=v_{x x}, \quad(x, t) \in \Omega_{T}:=\{(x, t): x>s(t), t \in(0, T)\} \\
v(s(t), t)=0 \\
v(\cdot, 0)=f^{\prime}
\end{array}\right.
$$

and assume that the free boundary $s$ satisfies $s \in C[0, T] \cap C^{1}(0, T]$. Integrating Green's identity

$$
\begin{gathered}
\frac{\partial}{\partial \xi}\left(\Gamma(x-\xi, t-\tau) v_{\xi}(\xi, \tau)-\frac{\partial}{\partial \xi} \Gamma(x-\xi, t-\tau) v(\xi, \tau)\right) \\
-\frac{\partial}{\partial \tau}(\Gamma(x-\xi, t-\tau) v(\xi, \tau))=0
\end{gathered}
$$


over the domain $\Omega_{t}$ we obtain, for $x>s(t)$, the representations

$$
v(x, t)=\int_{0}^{\infty} \Gamma(x-\xi, t) f^{\prime}(\xi) d \xi-\int_{0}^{t} \Gamma(x-s(\tau), t-\tau) v_{\xi}(s(\tau), \tau) d \tau
$$

$$
v_{x}(x, t)=\int_{0}^{\infty} \Gamma(x-\xi, t) f^{\prime \prime}(\xi) d \xi-\int_{0}^{t} \Gamma_{x}(x-s(\tau), t-\tau) v_{\xi}(s(\tau), \tau) d \tau
$$

Passing to the limit $x \rightarrow s(t)^{+}$in (13) yields

$$
r(t)=2 \int_{0}^{\infty} \Gamma(s(t)-\xi, t) f^{\prime \prime}(\xi) d \xi-2 \int_{0}^{t} \Gamma_{x}(s(t)-s(\tau), t-\tau) r(\tau) d \tau
$$

where (see (6) and (7)) $r(t)=d f(s(t)) / d t=v_{x}(s(t), t)$. The justification for this passage to the limit is contained in the following result.

LEMMA 1. If $s \in C([0, T]) \cap C^{1}((0, T])$ and $r \in C([0, T])$, we have for $t<T$

$$
\lim _{x \searrow s(t)} \int_{0}^{t}\left[\Gamma_{x}(s(t)-s(\tau), t-\tau)-\Gamma_{x}(x-s(\tau), t-\tau)\right] r(\tau) d \tau=\frac{1}{2} r(t) \text {. }
$$

Proof. We write

$$
\begin{aligned}
& \int_{0}^{t}[\cdots] r(\tau) d \tau=\frac{1}{4 \sqrt{\pi}} \int_{0}^{t} \frac{s(t)-s(\tau)}{(t-\tau)^{3 / 2}}\left[\exp \left(-\frac{(x-s(\tau))^{2}}{4(t-\tau)}\right)\right. \\
& +\frac{1}{4 \sqrt{\pi}} \int_{0}^{t} \frac{x-s(t)}{(t-\tau)^{3 / 2}}\left[\exp \left(-\frac{(s(t)-s(\tau))^{2}}{4(t-\tau)}\right)\right] r(\tau) d \tau \\
& \left.+\frac{1}{4 \sqrt{\pi}} \int_{0}^{t} \frac{x-s(t)}{(t-\tau)^{3 / 2}} \exp \left(-\frac{(x-s(\tau))^{2}}{4(t-\tau)}\right)-\exp \left(-\frac{(x-s(t))^{2}}{4(t-\tau)}\right)\right] r(\tau) d \tau
\end{aligned}
$$

In view of the assumptions on $s$ and $r$ it is easy to see that, for $\nu=1,2$,

$$
\left|\int_{0}^{t} I_{\nu}\right| \leqslant\left|\int_{t-\delta}^{t} I_{\nu}\right|+\left|\int_{0}^{t-\delta} I_{\nu}\right| \leqslant O(\sqrt{\delta})+c_{\delta} o(|x-s(t)|)
$$

which implies that

$$
\lim _{x \searrow s(t)} \int_{0}^{t} I_{\nu}=0, \quad \nu=1,2
$$

Finally,

$$
\frac{1}{4 \sqrt{\pi}} \int_{-\infty}^{t} \frac{y}{(t-\tau)^{3 / 2}} \exp \left(-\frac{y^{2}}{4(t-\tau)}\right) d \tau=\frac{1}{2}
$$

implies that

$$
\lim _{x \searrow s(t)} \int_{0}^{t} I_{3}=\frac{1}{2} r(t)
$$


3. Proof of the Theorem. We write the integral equation (14) in the form

$$
\begin{aligned}
r(t)= & \frac{1}{\sqrt{\pi}} \int_{0}^{\infty} \exp \left(-\frac{1}{4}\left(\frac{s(t)}{\sqrt{t}}-\xi\right)^{2}\right) f^{\prime \prime}(\xi \sqrt{t}) d \xi \\
& +\frac{1}{\sqrt{\pi}} \int_{0}^{1} \frac{A(s, t, \tau)}{1-\tau} \exp \left(-A(s, t, \tau)^{2}\right) r(t \tau) d \tau \\
= & :(F r)(t)+(K r)(t),
\end{aligned}
$$

where

$$
A(s, t, \tau):=\frac{s(t)-s(t \tau)}{2(t-t \tau)^{1 / 2}}
$$

It will be convenient to introduce the class of functions $\mathcal{H}^{\alpha}[0, T], 0<\alpha<1$, defined by

$$
\mathcal{H}^{\alpha}[0, T]=\left\{\rho:[0, T] \rightarrow \mathbf{R}:|\rho|_{\alpha}:=\sup _{0<t \leqslant T} t^{1-\alpha}\left|\rho^{\prime}(t)\right|<\infty\right\} .
$$

The class $\mathfrak{H}^{\alpha}$ is obviously contained in the Hölder-class with exponent $\alpha$.

The Theorem is a consequence of

Proposition. For any $\alpha \in(0,1 / 2)$, the integral equation (15), with s related to $r$ by (7), has a solution $r \in \mathcal{H}^{\alpha}[0, T]$ for some $T>0$. The constant $\kappa:=\sqrt{r(0)} / \beta$ $\left(\beta^{2}=\frac{1}{2} f^{\prime \prime}(0)\right)$ does not depend on $f$ and is implicitly determined by the equation

$$
\begin{aligned}
\frac{4}{\sqrt{\pi}} \int_{0}^{\infty} \exp (- & \left.\left(\frac{\kappa}{2}+\xi\right)^{2}\right) d \xi \\
& =\kappa^{2}\left(1+\frac{1}{\sqrt{\pi}} \int_{0}^{1} \frac{\kappa}{2} \frac{1}{\sqrt{1-\tau}(1+\sqrt{\tau})} \exp \left(-\frac{\kappa^{2}}{4} \frac{1-\sqrt{\tau}}{1+\sqrt{\tau}}\right) d \tau\right)
\end{aligned}
$$

the numerical value of $\kappa$ is $0.9034 \ldots$.

REMARK. The Proposition does not assert uniqueness of the function $r$ (hence of the free boundary $s$ ) which could be established by showing that the operator $F+K$ in (15) is a strict contraction; this is technically even more complicated than our proof. However, the uniqueness of $r$ is a consequence of the uniqueness of solutions of the original problem (1) discussed in the Introduction.

We prove the Proposition by iterating the integral equation (15) in the form

$$
r_{n+1}=F r_{n}+K r_{n}, \quad n \in \mathbf{N},
$$

with $r(0)=\kappa^{2} \beta^{2}$, where $\kappa$ is the solution of (16) and $\beta^{2}=\frac{1}{2} f^{\prime \prime}(0)$.

We shall show as a consequence of Lemmas 2 and 3 that, for $r \in \mathcal{H}^{\alpha}$ with $r(0)=\kappa^{2} \beta^{2}$

$$
\begin{gathered}
\lim _{t \rightarrow 0^{+}}(F r)(t)=\frac{1}{\sqrt{\pi}} \int_{0}^{\infty} \exp \left(-\frac{1}{4}(\kappa+\xi)^{2}\right) 2 \beta^{2} d \xi \\
\lim _{t \rightarrow 0^{+}}(K r)(t)=-\frac{1}{\sqrt{\pi}} \int_{0}^{1} \frac{\kappa}{2} \frac{1}{\sqrt{1-\tau}(1+\sqrt{\tau})} \exp \left(-\frac{\kappa^{2}}{4} \frac{1-\sqrt{\tau}}{1+\sqrt{\tau}}\right) \kappa^{2} \beta^{2} d \tau .
\end{gathered}
$$

Since $\kappa$ is the solution of (16), this implies that $r_{n}(0)=\kappa^{2} \beta^{2}$ for $n \in \mathbf{N}$. 
Moreover, we shall establish the a priori estimates: for $r \in \mathcal{H C}^{\alpha}[0, T], 0<\alpha<1 / 2$, (20)

$$
|F r|_{\alpha} \leqslant c(T)+\left(c_{1}(\alpha)+c(T)\right)|r|_{\alpha},
$$

where $c_{1}(\alpha)=(\kappa \sqrt{\pi}(1+\alpha))^{-1} \exp \left(-\frac{1}{4} \kappa^{2}\right)$, and

$$
|K r|_{\alpha} \leqslant\left(c_{2}(\alpha)+c(T)\right)|r|_{\alpha},
$$

where $c_{2}(\alpha)=c_{21}(\alpha)+c_{22}(\alpha)$ with

$$
\begin{aligned}
c_{21}(\alpha) & =\frac{\kappa}{2 \sqrt{\pi}} \int_{0}^{1} \frac{\tau^{\alpha}}{\sqrt{1-\tau}(1+\sqrt{\tau})} \exp \left(-\frac{\kappa^{2}}{4} \frac{1-\sqrt{\tau}}{1+\sqrt{\tau}}\right) d \tau, \\
c_{22}(\alpha)= & \frac{\kappa(1+1 /(2+2 \alpha))}{\sqrt{\pi}(2+4 \alpha)} \int_{0}^{1} \frac{1-\tau^{1 / 2+\alpha}}{(1-\tau)^{3 / 2}}\left(1-\frac{\kappa^{2}}{2} \frac{1-\sqrt{\tau}}{1+\sqrt{\tau}}\right) \\
& \times \exp \left(-\frac{\kappa^{2}}{4} \frac{1-\sqrt{\tau}}{1+\sqrt{\tau}}\right) d \tau,
\end{aligned}
$$

and where $c(T)$ is a constant such that $c(T) \rightarrow 0$ as $T \rightarrow 0^{+}$, uniformly for $r \in\{\rho$ : $|\rho(0)|+|\rho|_{\alpha} \leqslant$ const $\rangle$.

We first use the estimates (20), (21) to complete the proof of the Proposition. Combining the estimates (20) and (21) one has

$$
\left|r_{n+1}\right|_{\alpha} \leqslant c(T)+\left(c_{1}(\alpha)+c_{2}(\alpha)+c(T)\right)\left|r_{n}\right|_{\alpha} .
$$

Crucial for the following argument is the fact that

$$
c_{1}\left(\frac{1}{2}\right)+c_{2}\left(\frac{1}{2}\right)=0.339 \ldots+0.453 \ldots=: \omega<1 .
$$

Set $\bar{\omega}:=(1+\omega) / 2<1$ and choose $\alpha \in(0,1 / 2)$ close to $1 / 2$ and $T>0$ such that for all $r \in \mathcal{H}^{\alpha}$ with $r(0)=\kappa^{2} \beta^{2}$ and $|r|_{\alpha} \leqslant 1 /(1-\bar{\omega})$

$$
c_{1}(\alpha)+c_{2}(\alpha)+c(T) \leqslant \bar{\omega} .
$$

It should be observed that if one chooses $\alpha \geqslant 1 / 2$ then one cannot prove the crucial estimate (20), cf. e.g. (24). By (22), we have

$$
\left|r_{n}\right|_{\alpha} \leqslant 1 /(1-\bar{\omega}), \quad n \in \mathbf{N} .
$$

Hence we can select a subsequence of $r_{n}$ which converges in $C[0, T]$ to a function $r_{\infty} \in \mathcal{H}^{\alpha}[0, T]$ with $r_{\infty}(0)=\kappa^{2} \beta^{2}$. Set $s_{n}:=g\left(\sqrt{R_{n}}\right)$. To pass to the limit in (17) note that by Lemmas 2 and 3 the expressions $\exp \left(-\frac{1}{4}\left(s_{n}(t) / \sqrt{t}-\xi\right)^{2}\right)$ and $\left(A\left(s_{n}, t, \tau\right) /(1-\tau)\right) \exp \left(-A\left(s_{n}, t, \tau\right)^{2}\right)$ converge pointwise (for $\left.n \rightarrow \infty\right)$ and are majorized by integrable functions, uniformly in $n \in \mathbf{N}$. This completes the proof of the Proposition and the Theorem.

It remains to establish the assertions (18)-(21). We require two auxiliary results. We denote by $c$ a generic constant which may depend on $\alpha,|r|_{\alpha}$ and $T$, and we assume throughout that $T=T\left(|r|_{\alpha}, \alpha\right)$ is sufficiently small.

Lemma 2. For $r \in \mathcal{H}^{\alpha}, \alpha \in(0,1 / 2)$, with $r(0)=\kappa^{2} \beta^{2}$ we have

$$
|s(t)+\kappa \sqrt{t}| \leqslant c t^{1 / 2+\alpha} \text {. }
$$


Proof. Note that $|r(t)-r(0)| \leqslant c t^{\alpha}$ and therefore $|R(t)-r(0) t| \leqslant c t^{1+\alpha}$. Using (10), (11) and this inequality one has

$$
|s(t)+\kappa \sqrt{t}|=|g(\sqrt{R(t)})+\kappa \sqrt{t}| \leqslant\left|-\beta^{-1} \sqrt{R(t)}+\kappa \sqrt{t}\right|+c t \leqslant c t^{1 / 2+\alpha}+c t .
$$

LEMMA 3. For $r \in \mathcal{H}^{\alpha}$ with $r(0)=\kappa^{2} \beta^{2}$ we have

$$
|A(s, t, \tau)| \leqslant c \frac{1-\sqrt{\tau}}{\sqrt{1-\tau}}=c \frac{\sqrt{1-\tau}}{1+\sqrt{\tau}} .
$$

Proof. Using $f^{\prime}(s) s^{\prime}=r,(9)$ and Lemma 2, we obtain

$$
|s(t)-s(t \tau)|=\left|\int_{t \tau}^{t} \frac{r(\sigma)}{f^{\prime}(s(\sigma))} d \sigma\right| \leqslant c \int_{t \tau}^{t}\left(2 \beta^{2} \kappa \sqrt{\sigma}-c \sigma^{1 / 2+\alpha}\right)^{-1} d \sigma \leqslant c(\sqrt{t}-\sqrt{t \tau}) ;
$$

this establishes the claim by the definition of $A(s, t, \tau)$.

Lemma 3 shows that the kernel corresponding to the operator $K$ in (15) is integrable. Moreover, we see from Lemma 2 that

$$
A_{0}(\tau):=\lim _{t \rightarrow 0^{+}} A(s, t, \tau)=-\frac{\kappa}{2} \frac{1-\sqrt{\tau}}{\sqrt{1-\tau}} .
$$

Using this and Lemma 2, we can pass to the limit in (15), thus establishing (18) and (19).

Proof of (20). To estimate the norm of Fr, use the definition in (15) to form

$$
\begin{aligned}
\frac{d(F r)(t)}{d t} & =\frac{1}{\sqrt{\pi}} \int_{0}^{\infty} \exp \left(-\frac{1}{4}\left(\frac{s(t)}{\sqrt{t}}-\xi\right)^{2}\right) \frac{1}{2} t^{-1 / 2} \xi f^{\prime \prime \prime}(\xi \sqrt{t}) d \xi \\
& -\left[\frac{1}{\sqrt{\pi}} \int_{0}^{\infty} \frac{1}{2}\left(\frac{s(t)}{\sqrt{t}}-\xi\right) \exp \left(-\frac{1}{4}\left(\frac{s(t)}{\sqrt{t}}-\xi\right)^{2}\right) f^{\prime \prime}(\xi \sqrt{t}) d \xi\right] \times \frac{d}{d t} \frac{s(t)}{\sqrt{t}} .
\end{aligned}
$$

As $t \searrow 0$, the term in square brackets tends (use (9)) to

$$
\begin{aligned}
\frac{1}{\sqrt{\pi}} \int_{0}^{\infty} \frac{1}{2}(-\kappa-\xi) & \exp \left(-\frac{1}{4}(-\kappa-\xi)^{2}\right) 2 \beta^{2} d \xi \\
& =-\frac{2}{\sqrt{\pi}} \beta^{2} \exp \left(-\frac{1}{4} \kappa^{2}\right)=2(1+\alpha) \kappa \beta^{2} c_{1}(\alpha) .
\end{aligned}
$$

Therefore,

$$
\left|\frac{d(F r)(t)}{d t}\right| \leqslant c t^{-1 / 2}+\left(2(1+\alpha) \kappa \beta^{2} c_{1}(\alpha)+\bar{c}(t)\right)\left|\frac{d}{d t}\left(\frac{s(t)}{\sqrt{t}}\right)\right| .
$$

It remains to estimate $(d / d t)(s(t) / \sqrt{t})$. Using (7), Lemma 2, (9) and (10) we have

$$
\begin{aligned}
\left|\frac{s^{\prime}(t)}{\sqrt{t}}-\frac{1}{2} \frac{s(t)}{t^{3 / 2}}\right| & =t^{-3 / 2}\left|\frac{1}{f^{\prime}(s(t))}\right|\left|\operatorname{tr}(t)-\frac{1}{2} s(t) f^{\prime}(s(t))\right| \\
& \leqslant t^{-3 / 2} t^{-1 / 2}\left(\frac{1}{2} \beta^{-2} \kappa^{-1}+\bar{c}(t)\right)\left[\left|\operatorname{tr}(t)-\beta^{2} s(t)^{2}\right|+\bar{c} t^{3 / 2}\right] \\
& \leqslant t^{-1}\left(\frac{1}{2} \beta^{-2} \kappa^{-1}+\bar{c}(t)\right)\left[|\operatorname{tr}(t)-R(t)|+\bar{c} t^{3 / 2}\right] .
\end{aligned}
$$


A simple calculation shows that

$$
|\operatorname{tr}(t)-R(t)| \leqslant \frac{1}{1+\alpha} t^{1+\alpha}|r|_{\alpha}
$$

and this yields

$$
\left|\frac{d}{d t}\left(\frac{s(t)}{\sqrt{t}}\right)\right| \leqslant t^{\alpha-1}\left(\frac{1}{2} \beta^{-2} \kappa^{-1}+\bar{c}(t)\right)\left(\frac{1}{1+\alpha}+\bar{c}(t)\right)|r|_{\alpha} .
$$

Combining (24) and (26) proves (20).

We next turn to the proof of (21). We write (cf. (15))

$$
\begin{aligned}
\frac{d}{d t}(K r)(t)= & \frac{1}{\sqrt{\pi}} \int_{0}^{1} \frac{1}{1-\tau} A \exp \left(-A^{2}\right) \tau r^{\prime}(t \tau) d \tau \\
& +\frac{1}{\sqrt{\pi}} \int_{0}^{1} \frac{1}{1-\tau}\left(1-2 A^{2}\right) \exp \left(-A^{2}\right) \frac{d A}{d t} r(t \tau) d \tau \\
= & :\left(K_{1} r\right)(t)+\left(K_{2} r\right)(t)
\end{aligned}
$$

and estimate each term separately.

(i) Since $\left|r^{\prime}(t \tau)\right| \leqslant(t \tau)^{\alpha-1}|r|_{\alpha}$, it follows from (23) that

$$
\left|\left(K_{1} r\right)(t)\right| \leqslant\left(c_{21}+c(t)\right) t^{\alpha-1}|r|_{\alpha} .
$$

(ii) To estimate $K_{2} r$ we first consider the term $d A(s, t, \tau) / d t$. Using the definition of $A$ and (7), we obtain

$$
\begin{aligned}
2(t-t \tau)^{1 / 2} t \frac{d}{d t}\left(\frac{1}{2} \frac{s(t)-s(t \tau)}{(t-t \tau)^{1 / 2}}\right) \\
=t s^{\prime}(t)-(t \tau) s^{\prime}(t \tau)-\frac{1}{2} s(t)+\frac{1}{2} s(t \tau) \\
=\int_{t \tau}^{t} \frac{d}{d \sigma}\left(\sigma s^{\prime}(\sigma)-\frac{1}{2} s(\sigma)\right) d \sigma \\
=\int_{t \tau}^{t}\left(\frac{1}{2} s^{\prime}(\sigma)+\sigma \frac{d}{d \sigma}\left(\frac{r(\sigma)}{f^{\prime}(s(\sigma))}\right)\right) d \sigma
\end{aligned}
$$

i.e.

$$
\frac{d A}{d t}=\frac{1}{2} t^{-1}(t-t \tau)^{-1 / 2} \int_{t \tau}^{t}\left(Q_{1}(\sigma)+Q_{2}(\sigma)\right) d \sigma
$$

with

$$
\begin{aligned}
& Q_{1}(\sigma):=\sigma \frac{r^{\prime}(\sigma)}{f^{\prime}(s(\sigma))} \\
& Q_{2}(\sigma):=s^{\prime}(\sigma)\left(\frac{1}{2}-\sigma \frac{r(\sigma) f^{\prime \prime}(s(\sigma))}{f^{\prime}(s(\sigma))^{2}}\right) .
\end{aligned}
$$


We estimate each term separately. By Lemma 2 and (9), we have

$$
\begin{aligned}
\int_{t \tau}^{t}\left|Q_{1}(\sigma)\right| d \sigma & \leqslant \int_{t \tau}^{t} \sigma \frac{\sigma^{\alpha-1}|r|_{\alpha}}{2 \beta^{2} \kappa \sqrt{\sigma}-c \sigma^{1 / 2+\alpha}} d \sigma \\
& \leqslant\left(\frac{1}{2} \frac{1}{1 / 2+\alpha} \beta^{-2} \kappa^{-1}+c(t)\right) t^{1 / 2+\alpha}\left(1-\tau^{1 / 2+\alpha}\right)|r|_{\alpha} .
\end{aligned}
$$

We write $Q_{2}$ in the form

$$
Q_{2}(\sigma)=\frac{r(\sigma)}{f^{\prime}(s(\sigma))^{3}}\left(\frac{1}{2}\left(f^{\prime}(g(\sqrt{R(\sigma)}))\right)^{2}-\sigma r(\sigma) f^{\prime \prime}(s(\sigma))\right) .
$$

Since by (9), (10) and Lemma 2 ,

$$
\left.\begin{array}{l}
\left|\frac{1}{2}\left(f^{\prime}(g(\sqrt{R(\sigma)}))\right)^{2}-2 \beta^{2} R(\sigma)\right| \\
\left|\sigma r(\sigma) f^{\prime \prime}(s(\sigma))-2 \beta^{2} \sigma r(\sigma)\right|
\end{array}\right\} \leqslant c \sigma^{3 / 2},
$$

we obtain, also using (25),

(30)

$$
\begin{aligned}
\int_{t \tau}^{t}\left|Q_{2}(\sigma)\right| d \sigma & \leqslant \int_{t \tau}^{t} \frac{r(0)}{\left(2 \beta^{2} \kappa \sqrt{\sigma}\right)^{3}}(1+c(\sigma))\left(2 \beta^{2} \frac{1}{1+\alpha} \sigma^{1+\alpha}|r|_{\alpha}\right) d \sigma \\
& \leqslant\left(\frac{1}{4} \frac{1}{(1+\alpha)(1 / 2+\alpha)} \beta^{-2} \kappa^{-1}+c(t)\right) t^{1 / 2+\alpha}\left(1-\tau^{1 / 2+\alpha}\right)|r|_{\alpha} .
\end{aligned}
$$

Combining (29) and (30) with (28), it follows that

$$
\begin{aligned}
\left|\left(K_{2} r\right)(t)\right| \leqslant & \frac{1}{4} \frac{1}{1 / 2+\alpha} \beta^{-2} \kappa^{-1}\left(1+\frac{1}{2} \frac{1}{1+\alpha}\right)(1+c(t)) t^{\alpha-1}|r|_{\alpha} \\
& \times \frac{1}{\sqrt{\pi}} \int_{0}^{1} \frac{1-\tau^{1 / 2+\alpha}}{(1-\tau)^{3 / 2}}\left(1-2 A_{0}(\tau)^{2}\right) \exp \left(-A_{0}(\tau)^{2}\right)(1+c(t)) r(0) d \tau .
\end{aligned}
$$

Adding the estimates (27) and (31) proves (21).

REMARK. We conjecture that, for smooth initial data $f$, the function $r\left(t^{2}\right)$ is smooth, i.e.

$$
r(t)=\kappa^{2} \beta^{2}+r_{1 / 2} \sqrt{t}+r_{1} t+\ldots
$$

Assuming an expansion of the form (32), we can calculate the coefficients $r_{1 / 2}, r_{1}, \ldots$ from the integral equation (15). In particular, $f^{\prime \prime \prime}(0) \neq 0$ implies that $r_{1 / 2} \neq 0$. This shows that (8) is, in general, not valid for $\alpha>1 / 2$.

\section{REFERENCES}

1. P. Benilan, M. G. Crandall and A. Pazy, M-accretive operators (in preparation).

2. L. C. Evans, Application of nonlinear semigroup theory to certain partial differential equations, Nonlinear Evolution Equations (M. G. Crandall, ed.), Academic Press, New York, 1978.

3. A. Fasano and M. Primicerio, General free boundary problems for the heat equation. I, J. Math. Anal. Appl. 57 (1977), 694-723.

4. 202-231. 
5. K. Höllig, Existence of infinitely many solutions for a forward backward heat equation, Trans. Amer. Math. Soc. 278 (1983), 299-316.

6. K. Höllig and J. A. Nohel, A diffusion equation with a nonmonotone constitutive function, Systems of Partial Differential Equations (J. M. Ball, ed.), Reidel, Dordrecht, 1983, pp. 409-422.

7. D. Kinderlehrer and L. Nirenberg, Regularity in free boundary problems, Ann. Scuola Norm. Sup. Pisa Cl. Sci. (4) 4 (1977), 373-391.

8. D. Schaeffer, A new proof of the infinite differentiability of the free boundary in the Stefan problem, J. Differential Equations 20 (1976), 266-269.

Mathematics Research Center, University of Wisconsin, Madison, Wisconsin 53706 